\title{
Micromycetes (Peronosporaceae, Erysiphales and Uredinales) new to the province of Kuusamo, N. E. Finland
}

\author{
Teuvo Ahti
}

Botanical Museum, University of Helsinki

The detailed distribution of the species of most groups of micromycetes in Finland is rather poorly known. This is particularly true with central and northern Finland. During an inventory of the anthropochorous flora of vascular plants in the biogeographical province of Kuusamo, N. E. Finland, the writer also collected fungi and made field records on their occurrence. The present list includes the species and host combinations of the parasitic groups Peronosporaceae, Erysiphales and Uredinales that appear to be new to the province according to literature.

Kuusamo consists largely of an upland district, about 200 to $400 \mathrm{~m}$ in altitude, forming a southward extension of the northern boreal bioclimatic vegetation zone. The growing season is fairly humid, approaching distinctly oceanic conditions, while at the same time it is rather continental in the thermal sense. A number of species of parasitic fungi have ranges different from those of their hosts (e.g. Jörstad 1964 b), and it may be noted that some species common in southern Finland (e.g. Erysiphe galeopsidis, Uromyces fallens, Puccinia graminis) are absent or scarce in Kuusamo, although suitable hosts are abundant. On the other hand, some species common in Lapland are frequent (e.g. Uromyces sommerfeltii, Puccinia deschampsiae, $P$. poaenemoralis on Anthoxanthum odoratum). In general, some temperate to boreal spe- cies have northern and some arctic-alpine species southern outposts in Kuusamo. It seems clear that species of Peronospora and Erysiphales are fewer and less abundant in northern than in southern Finland (cf. Gustavsson 1959; MäkINEN 1963). In addition to the effects of climate, the number of species is restricted by the fact that agricultural landscape covers only a minor part of the northern areas, which reduces the number of hosts and frequency of suitable habitats.

In the present paper the species of Peronospora are named according to Gustavsson (1959), with his very narrow species concept, while in the rest of Peronosporaceae JörSTAD (1964 a) is followed. The species are considered as new to the province if not included in the summarizing papers by Gustavsson (op. c.) and Rauhala (1966), who recorded only 7 species from the province.

Erysiphales are treated according to Blumer (1933), with the exception of the Sphaerotheca fuliginea complex, which is according to JunELL (1966). The nomenclatural changes proposed by her (JunELL 1965) have also been adopted. Only 5 species of Erysiphales were reported from Kuusamo by Rauhala (1957) and MäKINEN (1965).

In Uredinales the wide species concept of Hylander, Jörstad \& NANNFELdT (1953) is followed. A few changes in their nomen- 
clature have been adopted from the treatments of Jörstad (1960, 1962) and WilsoN \& Henderson (1966). The species are considered as new to Kuusamo (or to Finland) if not included in the papers by RauHaLA (1959) and Mäkinen (1964 a, 1964 b). They recorded 78 host-fungus combinations for Kuusamo (many of them so far only known from the present Soviet part of Kuusamo, however).

The nomenclature of vascular plants is chiefly in accordance with HyLANDER (1955), with some exceptions.

The specimens are deposited in Botanical Museum, University of Helsinki (H), unless otherwise indicated. Two specimens are in the herbaria of the Universities of Oulu (OULU) and Turku (TUR). Dr. Heikki Roivainen, Helsinki, and Dr. Ivar Jörstad, Oslo, have kindly confirmed some identifications.

Abbreviations:

$$
\begin{aligned}
\text { T.A. } & =\text { Teuvo Ahti } \\
\text { L.H.A. } & =\text { Leena Hämet-Ahti } \\
\text { J.V. } & =\text { Juha Viramo } \\
\mathrm{s} & =\text { sight record } \\
* & =\text { species new to the province of } \\
& \text { Kuusamo } \\
\mathrm{K} & =\text { Kuusamo parish } \\
\mathrm{P} & =\text { Posio parish } \\
\mathrm{T} & =\text { Taivalkoski parish } \\
\mathrm{Kk} & =\text { parish centre (kirkonkylä) }
\end{aligned}
$$

\section{Peronosporaceae}

Albugo candida (Pers.) O. Kuntze - On Erysimum cheiranthoides ssp. altum. K: Kk, 3 locs. 1965 and 1966 T.A. In northern Finland the species is rather frequent on ssp. altum Ahti. MäKINEN \& HiETAJäRvi (1965) reported $A$. candida on Erysimum hieraciifolium from one Finnish locality (Petolahti) according to the present writer's specimen. However, the specimen belongs to $E$. cheiranthoides ssp. altum (see Aнtr 1962, p. 30).

*Bremia lactucae Regel - On Carduus crispus. K: Särkiluoma 1966 T.A.

*Peronospora alsinearum Casp. - On Stellaria media. K: Mattila 1966 T.A.; Yli-Suininki 1966 T.A.

* P. alta Fuck. - On Plantago major. K: Kk 1966 T.A.

*P. mayorii Gäum. - On Vicia cracca. K: Kk, 4 locs. 1966 T.A. (3 s); Särkelä 1966 T.A.

*P. parasitica (Fr.) Fr. - On Capsella bursa-pastoris. K: Kk 1966 T.A.

*P. polygoni Thüm. ex A. Fisch. - On Polygonum aviculare s. str. (P. aviculare ssp. heterophyllum). K: Salminen 1966 T.A.

P. ranunculi Gäum. - On Ranunculus acris. K: Kk 1965 T.A.
* $P$. sepium Gäum. - On Vicia sepium var. sepium. K: Kk, Kiviharju 1965 T.A. - On V. sepium var. montana. K: Kk 1966 T.A.; Murtovaara 1966 T.A.; Paanajärvi, Päätalo 1928 Eva Alander (H).

* Plasmopara crustosa (Fr.) Jörst. (P. nivea auct.) - On Angelica sylvestris. K: Kk, centre and Saunalainen 1966 T.A.; Kärpänkylä 1966 T.A.; Penttilänvaara 1966 T.A. (s). - On Anthriscus sylvestris. K: Kk 1966 T.A. (s); Vanttaja, Sänkikangas, Ojala 1966 T.A.

*P. pusilla (de Bary) Schroet. - On Geranium sylvaticum. K: Penttilänvaara 1966 T.A.

\section{Erysiphales}

*Erysiphe asperifoliorum Grev. - On Myosotis arvensis. K: Rukajärvi, 1949 E. Laaksonen (TUR).

*E. cichoracearum Mérat - On Hieracium (group Piloselloidea) sp. K: Kk 1966 T.A. - On Hieracium (group Vulgata) spp. K: Kärpänkylä, Koppelo T.A.; Vuotunki 1966 T.A. (s). P: Suonnankylä 1966 T.A.

*E. graminis Mérat - On Agropyron (Elytrigia) repens. K: Kk 1965 T.A. In 1966 extremely common on this host in $\mathrm{K}$ and $\mathrm{P}$, probably seen in every one of the about 60 villages visited. - On Festuca rubra. K: Kk, centre and Petäjälampi 1966 T.A. Not reported on this host in Finland before. - On Poa pratensis ssp. alpigena. K: Kk 1966 T.A. (s). Probably very common.

E. ranunculi Grev. - Aconitum $\times$ cammarum. K: Kk 1966 T.A.; Tuovila 1966 T.A. Not reported on this host in Finland before. - On Ranunculus acris coll. K: Kk 1966 T.A.; Koskenkylä, Jämsä 1966 T.A.

E. trifolii Grev. - On Lathyrus pratensis. K: Kk, two locs. by Petäjälampi 1966 T.A. - On Trifolium hybridum. K: Kk 1965 T.A.

*Podosphaera myrtillina (Schub.) Kuntze \& Schmidt - On Vaccinium myrtillus. K: Oivanki, Rantalahti 1966 T.A. - On V. vitis-idaea. K: Rukatunturi 1964 L.H-A. Not reported on this host in Finland before. Blumer (1933) reported only Podosphaera major (Juel) Blum. on this host.

Sphaerotheca alchemillae (Grev.) L. Junell - On Alchemilla spp. (incl. micans, glomerulans, subcrenata). K: Kk, several locs. 1965 and 1966 T.A.; Määttälänvaara 1966 T.A.; Tammela 1966 T.A.; Tuovila 1966 T.A.; Oivanki 1966 T.A. (s); Säkkilänvaara 1966 T.A.; Kuoliovaara 1966 T.A.; Penttilänvaara 1966 T.A. Very common and abundant on Alchemilla species, which are present in most villages.

*S. fugax Penz. \& Sacc. - On Geranium sylvaticum. K: Virranniemi 1966 T.A.; Säkkilänvaara 1966 T.A.

*S. fuliginea (Fr.) Poll. s. str. - On Veronica longifolia. K: Kk, 1966 T.A.

* S. melampyri L. Junell - On Melampyrum sylvaticum. Liikasenvaara 1966 T.A.

\section{Uredinales}

Melampsora epitea Thüm. This is an extremely common species in the area and therefore was not especially looked for. To the four hosts reported by MäkINEN (1964) for Kuusamo the following ones may be added. - On Salix aurita $\times$ myrtilloides. K: Kk, Säynäjävaara 1965 T.A. - On S. borealis. 
K: Kk 1965 T.A. (s). - On S. caprea. K: Kk 1965 T.A. - On S. lapponum. K: Kk 1965 T.A.; Oivanki, Rantalahti 1966 T.A. (s). - On S. myrsinifolia s. str. K: Kk 1965 T.A. - On S. starkeana s. str. K: Lämsänkylä 1966 T.A.; Käylä W, Rävä 1966 T.A. - On S. xerophila. K: Kk, Nilonkangas and Kolvanki 1966 T.A.

* Puccinia acetoase Körn. - On Rumex acetoca ssp. acetosa. K: Kk 1965 T.A.; Riekki 1966 T.A. On R. tenuifolius. K: Kk 1963 T.A.

$P$. calcitrapae DC. - On Cirsium heterophyllum. K: Kk 1965 and 1966 T.A.; Määttälänvaara 1966 T.A.; Haataja 1966 T.A.

$P$. caricina DG. var. uliginosa (Juel) Jörst. - On Carex nigra s. lat. K: Kk 1966 T.A.

$P$. caricina DG. var. urticae-acutae (Kleb.) Hend. - On Carex aquatilis. K: Kk 1966 T.A. Host new to Finland. - On Urtica dioeca ssp. dioeca. $\mathrm{K}$ : Kk, several locs. 1965 and 1966 T.A.

*P. coronata Corda - On Agrostis borealis. K: Kk 1966 T.A.; Kemilä, Kuokkalampi 1966 T.A.; Kuparivaara, $4 \mathrm{~km} \mathrm{~W}$. of Poussunjärvi 1966 T.A. On $A$. tenuis. K: Kuparivaara, as above 1966 T.A. - No rusts have been reported on Agrostis borealis in Finland before. Only uredospores were present, but the identification was confirmed by Dr. I. Jörstad. The aecidial host Rhamnus frangula was not present in the vicinity (very rare in Kuusamo) but Jörstad (1964 b, p. 125) and MäKInEN (1964 a, p. 166) have suggested that $P$. coronata may be independent on host alternation in northern Norway and Finland.

*P. deschampsiae Arth. - On Deschampsia caespitosa. K: Kk, Kitron Park 1965 'T.A.; Penttilänvaara 1966 T.A.; Suininki, Lehto 1966 T.A.; Virranniemi 1966 T.A.

$P$. dioicae P. Magn. - On Carex ericetorum. $\mathrm{K}$ : Kk, Petäjälampi 1966 T.A.

P. fergussonii Berk. \& Br. - On Viola palustris. K: Kk, Säynäjäjoki 1965 T.A.; Vasaraperä 1966. T.A.

$P$. hieracii Mart. var. hieracii - On Leontodon autumnalis. K: Kk, 1966 T.A.; Mäkelä 1966 T.A.; Särkelä 1966 T.A.; Irni 1966 T.A.; Rukajärvi, Aikkila 1966 T.A.; Vasaraperä 1966 T.A.

$P$. hieracii Mart. *var. piloselloidarum (Probst) Jörst. - On Hieracium (group Piloselloidea) spp. K: Kk, Petäjälampi 1966 T.A.; Särkelä 1966 T.A.

* P. obscura Schroet. - On Luzula multiflora ssp. frigida. K: Kk, Petäjälampi 1966 T.A. Not earlier reported on this subspecies in Finland. The northernmost locality on L. multiflora s. lat. has been in Laukaa. North Häme (MÄknNEN 1964 b). Even in Norway only twice recorded on ssp. frigida (JöRSTAD \& GJAERUM 1964, p. 64).
* $P$. poarum Niels. - On Tussilago farfara. K: Liikasenvaara 1965 L.H-A.

*P. poae-nemoralis Otth - On Anthoxanthum odoratum (ssp. alpinum?). K: Kk, Nilonkangas and Petäjälampi 1966 T.A.; Kuoliovaara 1966 T.A.; Oivanki 1966 T.A.; Haataja 1966 T.A.; Törmäsenvaara 1966 T.A.; Sossonniemi 1966 T.A.; Särkiluoma 1966 T.A. P: Tolva 1966 T.A. - On Poa pratensis ssp. alpigena K: Kk. 1963 T.A. (very common); Mäkelä 1966 T.A.; Kurvinen E, Korpijärvi 1965 T.A. - On Poa pratensis ssp. pratensis. $\mathrm{K}$ : Kk, Nilonkangas and Petäjälampi 1963' and 1966 'T.A. (in 1966 almost constant on this host in Kk); Vanttaja, lumber camp $8 \mathrm{~km} \mathrm{~S}$. W. of Sänkikangas 1966 T.A.

*P. punctata Link - On Galium mollugo. Säkkilänvaara 1966 T.A.

$P$. recondita Desm. - On Agropyron (Elytrigia) repens. K: Kärpänkylä 1966 T.A. This race $(=P$. agropyrina Eriks.) of $P$. recondita has probably not been reported from Finland before. The identification of the present specimen (uredo stage) was confirmed by Dr. I. Jörstad. $P$. recondita on Agropyron repens is said to be common e.g. in Norway (JöRSTAD 1962, p. 108) and Britain (WrLson \& HeNDERSON 1966, p. 280) and has been certainly overlooked in Finland.

*P. thlaspeos Schub. - On Erysimum hieraciifolium. K: Oulankajoki, Hepokoski 1966 J.V. (OU$\mathrm{LU})$.

$P$. vaginatae Juel - On Carex vaginata. K: Jyrkänkoski 1966 T.A. New to present Finland but earlier found on the Soviet side of Kuusamo.

Pucciniastrum vaccinii (Wint.) Jörst. - On Vaccinium vitis-idaea. K: Kk, Petäjälampi 1966, T.A.; Oivanki, Rantalahti 1966 T.A.

*Uredo festucae DC. - On Festuca ovina. K: Kk 1965 T.A. - On F. rubra. K: Kk, Petäjälampi 1966 T.A.

*Uromyces airae-flexuosae Ferd. \& Winge - On Deschampsia flexuosa. K: Kurvinen, Korpijärvi 1965 T.A.; Heikkilänkylä 1966 T.A.; Vanttaja, 9 km S.W. of Sänkikangas 1966 T.A. Probably very common.

$U$. lapponicus Lagerh. - On Astragalus alpinus. K: Kiutaköngäs 1966 T.A. \& J.V.

U. polygoni-aviculariae (Pers.) Karst. - On Polygonum arenastrum ( $P$. aviculare ssp. aequale). $\mathrm{K}$ : Koskenkylä, Jämsä 1966 T.A. T: Kk 1963 T.A. RAUHALA (1957) reported this rust from the parish centres of Kuusamo and Salla on Polygonum aviculare coll., which probably means $P$. aviculare s. str. ( $P$. aviculare ssp. heterophyllum), a species that is much more abundant than $P$. arenastrum in Kuusamo.

\section{SUMMARY}

13 parasite-host combinations of Peronosporaceae, 16 of Erysiphales and 35 of Uredinales are reported as new to Kuusamo. The following combinations are new to Finland: Erysiphe graminis on Festuca rubra, E. ranunculi on Aconitum $\times$ cammarum, Podosphaera myrtillina on Vaccinium vitis-idaea, Puccinia caricina var. urticaeacutae on Carex aquatilis, $P$. coronata on Agrostis borealis, P. obscura on Luzula multiflora ssp. frigida, $P$. recondita on Agropyron repens, and $P$. vaginatae on Carex vaginata. 
Arti, T., 1962: On the taxonomy of Erysimum cheiranthoides L. (Cruciferae). - Arch. Soc. 'Vanamo' 16; 22-35.

Blumer, S., 1933: Die Erysiphaceen Mitteleuropas mit besonderer Berücksichtigung der Schweiz. - Beitr. Kryptog.fl. der Schweiz 7:1, 1-483.

Gustavssok, A., 1959: Studies on Nordic Peronosporas. I. Taxonomic revision. - Opera Bot. $3: 1,1-271$.

Hylander, N., 1955: Förteckning över Skandinaviens växter. I. Kärlväxter. Ed. 4. $175 \mathrm{pp}$. Lund.

Hylander, N., I. Jörstad \& J. A. Nannfeldt: Enumeratio Uredinearum Scandinavicarum. Opera Bot. 1:1, 1-102.

JörstaD, I., 1950: The graminicolous rust fungi of Norway. - Skr. Norske Vidensk.-Akad., Mat.Naturvetensk. K1. 1950:3, 1-92.

- 1960: The Norwegian rust species arranged alphabetically after host families and host genera, with short descriptions, mainly of macroscopical characters. - Nytt Mag. Bot. 8, 103-146.

- 1962: Distribution of the Uredinales within Norway. - Ibid. 9, 61-134.

- $1964 \mathrm{a}$ : The phycomycetous genera Albugo, Bremia, Plasmopara and Pseudoperonospora in Norway, with an appendix containing unpublished finds of Peronospora. - Ibid. 11, 47-82.

- $1964 \mathrm{~b}$ : The distribution within Norway of rust fungi (Uredinales) compared with the distribution of their hosts. - Ibid. 11, 109150.

Jörstad, I. \& H. B. GJaerum, 1964: Recent Norwegian finds of Uredinales and Ustilaginales. - Ibid. 12, 55-77.
Junell, Lena, 1965: Nomenclatural remarks on some species of Erysiphaceae. - Trans. Brit. mycol. Soc. $48,539-548$.

- 1966: A revision of Sphaerotheca fuliginea ([Schlecht.] Fr.) Poll. s. lat. - Svensk Bot. Tidskr. 60, 365-392.

Mäkrnen, Y., 1963: A tentitave list of Erysiphales in Inari Lapland (Finland). 6 pp. (mimeographed). Turku.

- 1964 a: On Finnish micromycetes 3. Uredinales of Inari Lapland. - Ann. Univ. Turkuensis A II:32, 155-177.

- 1964 b: On Finnish micromycetes. 4. On the distribution of rusts in Finland. - Ann. Bot. Fenn. 1, 214-219.

- 1965: On Finnish micromycetes. 7. Recent additions to the distribution of powdery mildews in Finland. - Ibid. 2, 243-247.

Mäkinen, Y. \& Lirsa Hietajärvi, 1965: On Finnish micromycetes. 5. Albugo candida in Finland, with special reference to the variation in the size of the conidia. - Ibid. 2, 33-46.

Rauhala, A., 1957: Mehltaupilzfunde aus Finnland mit Berücksichtigung der bisherigen Verbreitungsangaben. - Karstenia 4, 14-26.

- 1959: Luettelo Suomen ruostesienistä ja tietoja niiden kasvimaakunnittaisesta levinneisyydestä Itä-Fennoskandiassa. (Enumeratio Uredinearum Fennicarum et distributio hucusque cognita earum in provinciis phytogeographicis Fennoscandiae orientalis). Kuopion Luonn. Yst. Yhd. Julk. B 3:3, 1181.

- 1966: Mikrosienilöytöjä I. (Micromycetenfunde I). - Lahden Luonnonyst. Julk. 1, $1-26$.

Wilson, M. \& D. M. Henderson, 1966: British rust fungi. 384 pp. Cambridge.

Received 20.5. 1967. 